\title{
Species Index
}

Page numbers in italics refer to the subject of the papers

Achillea millefolium, 42

Adalia bipunctata, 423

Aegilops speltoides, 19

Agropyron pungens, 396

Allium montanum, 218

schoenoprasum, 83-87

Alnus glutinosa, 136

Amauris niavius, 422

Anthoxanthum odoratum, 37-45

Asclepias fruticosa, 191

Avena barbata, 7

longiglumis, 218

maroccana, 209-219

pilosa, 218

sativa, 209, 218

strigosa, 218

Bertholletis excelsa, 136

Beta maritima, 395-400

Biston betularia, 423

Bonamia ostreae, 369

Brassica alboglabra, 309-314

campestris, 309-314

napus, $309_{-314}$

oleracea, 89-95, 310, 314

Bulnesia, 321, 325, 326.

arborea, 326

bonariensis, 325,326

chilensis, 326

foliosa, 325, 326

retama, 326

sarmientoi, 326

schickendantzii, 325, 326

Camellia japonica, 136

Capsicum, 164

Carduus acanthoides, 329-337

nutans, 329-337

Catostomus clarkii, 258

Cepaea, 423

Chenopodium quinona, 16

Chlamydomonas, 151

Chondrina clienta, 239-244

Clintonia borealis, 42

Colias, 191, 193

meadii, 258

Coreopsis spp., 16

Cortaderia, 373-382

richardii, 373-382

selloana, 373-382

splendens, 380,381

toetoe, 379

Crab apple, 164

Crassostrea gigas, 359 virginica, 359, 368, 369, 370

Crataegus spp., 245-266

brachyacantha, 257

douglasii, 257

mollis, 246, 257

monogyna, 257

punctata, 257

Crepis capillaris, 19-27
Cyanococcus spp., 11, 17

Cynosurus cristatus, 181, 389

Danaus chrysippus, 409, 421, 422

plexippus, 191-194

Dendrocoelum lacteum, 97-106

Deroceras laeve, 7

Drosophila spp., 7, 57, 67, 156, 162, 267, 401

ananassae, 67-72

americana americana, 29-35

arizonensis, 29-35

auraria, 29-35

bipectinata, 67-72

borealis, 29-35

buzzatii, 156, 161

eohydei, 29-35

ercepeae, 67-72

ercepeae-like, 67-72

equinoxialis, 29-35

erecta, 29-35

hydei, 29-35

jambulina, 29-35

lachaisei, 67-72

lacteum, 104, 105

malerkotliana, 67-72

mauritiana, 155-162

melanogaster, $7,29-35,47-58,67,107-117,125-133,155$

$162,195-202,203-208,221-229,258,343-352,360,401$

mercatorum, 29-35

mojavensis, 29-35

monieri, 67-72

montana, 7, 29-35

mulleri, 29-35

nova-mexicana, 236

obscura, 67

orena, 29-35

parabipectinata, 67-72

paulistorum, 29-35

peninsularis, 29-35

persimilis, 231-237

pesudoananassae, 67-72

pseudoobscura, 199, 231-237

bogotana, 231-237

pseudoobscura, 231-237

repleta, 29-35

sechellia, 155-162

serido, 156, 161

serrata, 29-35

simulans, 29-35, 55, 155-162, 203

teissieri, 29-35

tropicalis, 29-35

vallismaia, 67-72

varians, $67-72$

virilis, 29-35

willistoni, 29-35, 55

yakuba, 29-35

Eucalyptus spp., 137

crucis, 390

delegatensis, 390

kitsoniana, 390

pauciflora, 390

regnans, 390 
Eucalyptus (continued) rhodantha, 383-393 stoatei, 384

Fagus sylvatica, 136

Fragaria $\times$ ananassa, 12

Fundulus heteroclitus, 258, 360, 370

Gaylussacia spp, 259

Haplopappus gracilis, 19 spinulosus, 12

Heuchera grossulariifolia, 12, 15 micrantha, 12, 15

Hordeum spontaneum, 7 vulgaris, 7

Hymenoptera, 59-65

Hypochoeris maculata, 25

Hypolimnas bolina, 420, 421 misippus, 409-425

Impatiens capensis, 1-9, 181-189 glandulifera, 150 pallida, 181-189

Indian mustard, 164

Ipomoea purpurea, 181

Isotoma petraea, 391

Juglans spp., 136

Larrea, 321-328

ameghinoi, 321, 323

cuneifolia, 321-328

divaricata, 321-328

nitida, 321-328

tridentata, 321-328

Liatris cylindracea, 181, 360, 370

Limnanthes douglasii, 373

Liriodendron tulipifera, 136

Locusta migratoria, 105

Lolium, 209, 218

multiflorum, 339-342

perenne, 42, 217, 218, 339-342 temulentum, 217

Lupinus succulentus, 7

Lycopersicon, 107

Macoma balthica, 359, 360, 370

maize, 164

Malus angustifolia, 246

coronaria, 246

diversifolia, 246

glabrata, 246

ioensis, 246

pumila, 245-266

Medicago spp., 16, 145-15.3

sativa, 12,146

Mercenaria mercenaria, 369

Mesostoma ehrenbergii ehrenbergii, 101, 104

Mulinia lateralis, 359, 369, 370

Mus musculus, 289-297, 299-308

ssp. bactrianus, 289-297, 299-308

castaneus, 289-297, 299--308

domesticus, 289-297, 299-308

mollossinus, 289-297, 299-308

musculus, 289-297, 299-308

Myrmeleotettix maculatus, 26, 86

Mytilus edulis, 359, 368, 369, 370
Nicotiana spp., 163, 164, 168

Oenothera, 145-153

Origanum vulgare, 395

Oryza glaberrima, 353-357

longistaminata, 356

rufipogon, 356

sativa, 163-170, 171-179, 353-357

Ostrea edulis, 359-372

Papillio glaucus, 259, 420

pea, 164

Pelargonium, 145-153

Petunia, 146, 164

hybrida, 146

parodii, 146

Pimephales promelas, 258

Pintoa, 321, 326, 327

chilensis, 326, 327

Pinus contorta, 181

pinaster, 267-274

resinosus, 242

Placopecten magellanicus, 359, 360, 370

Plantago lanceolata, 378, 379, 382, 395, 396, 399

Pleuronectes platessa, 360

Plumbago zeylanica, 150

Populus, 136

Potentilla glandulosa, 42

Primula, 423

Prosopis ruscifolia, 136

Pseudococcus affinis, 25

Psychotria nervosa, 181

Quercus petraea, 136

rapeseed, 164

Rattus fuscipes, 86

Rhagoletis mendax, 259 pomonella, 245-266

Rumina decollata, 242

Schistocerca gregaria, 105

Scilla, 209

Solanum spp., 16 tuberosum, 12

Spisula solidissima, 369

Thymus vulgaris, 395, 399

Tolmeia spp., 16

menziesii, 12

tomato, 164, 402

Tragopogon mirus, 12

miscellus, 12

Trifolium subterraneum, 37

Triticum spp, 299 aestivum, 12, 16, 218

Triturus alpetris, 119-123

ssp. alpestris, 119-123

cyreni, 119-123

Ulmus spp., 136

Urginia maritima, 16

Vaccinium spp, 16, 17, 259

corymbosum, 11-18

Wheat, 218

Zoarces viviparus, 140 